\title{
Estudio de seguimiento a personas graduadas de postgrados de la Facultad de Ciencias Sociales y Humanísticas (FCSH) de la Escuela Superior Politécnica del Litoral (ESPOL)
}

\section{Former graduate students follow-up of the Graduate Department of the Social Sciences and Humanities School (FCSH) at Escuela Superior Politécnica del Litoral (ESPOL)}

\author{
Katia Lorena Rodríguez-Morales ${ }^{1}$ \\ Escuela Superior Politécnica del Litoral (ESPOL) \\ Departamento de Postgrado de la Facultad de Ciencia Sociales y Humanísticas \\ Guayaquil, Ecuador \\ krodrig@espol.edu.ec \\ ORCID: 0000-0002-7613-2582 \\ Mónica Giomary Valenzuela-Ortiz² \\ Escuela Superior Politécnica del Litoral \\ Departamento de Postgrado de la Facultad de Ciencia Sociales y Humanísticas \\ Guayaquil, Ecuador \\ mogivale@espol.edu.ec \\ ORCID: $\underline{\text { 0000-0002-1839-5253 }}$
}

Recibido: 7 enero 2015 Aceptado: 16 junio 2016 Corregido: 21 julio 2016

1 Ecuatoriana, Ph.D. en Business Administration de Newport University, Magíster en Finanzas de la Universidad de Chile, Economista de la Escuela Superior Politécnica del Litoral (ESPOL). Cuenta con estudios de especialización en Finanzas en Georgetown University (Washington D.C.). Ha tomado cursos de formación profesional en INCAE, UCLA, USF y Shanghai Jiao Tong University. Posee experiencia docente a nivel de pregrado y postgrado en la Escuela Superior Politécnica del Litoral (ESPOL), en la Universidad de Especialidades Espíritu Santo (UEES), Universidad Católica de Santiago de Guayaquil (UCSG) en cursos de Teoría de Inversiones, Finanzas Corporativas, Finanzas II, Ingeniería Económica I y II, Habilidades Gerenciales, Principios de la Educación Superior, entre otros. Actualmente es docente a tiempo completo de la Facultad de Ciencias Sociales y coordina el programa la Maestría en Finanzas de la ESPOL. Colabora de forma activa con los organismos de control de educación superior de su país, en la revolución educativa que se ha venido llevando a cabo en los últimos años. Su última publicación es Modelo de cuantificación del impacto de la falsificación de productos en el valor de la empresa, publicado por la Revista Investigaciones Europeas de Economía y Dirección de Empresas. http:// econpapers.repec.org/article/idijiedee/v_3a20_3ay_3a2014_3ai_3a2_3ap_3a95-101.htm

2 Estudiante de la carrera de Ingeniería Comercial y Empresarial en la ESPOL, graduada del Centro Ecuatoriano Norteamericano en el año 2013 con un certificado de suficiencia de Inglés Intermedio. Además, aprobó todos los cursos de inglés en la Academia Copol English Institute. Actualmente labora como ayudante académica en el Departamento de Postgrados de la Facultad de Ciencias Sociales Humanísticas de la ESPOL. 
amplía la información con la que se contaba sobre este grupo de ex-estudiantes, ayudando así a tomar medidas y plantear recomendaciones que contribuirán a mantener los procesos de mejora continua de la unidad académica.

Palabras clave: Seguimiento a personas graduadas, estudios de postgrado, sujetos graduados de postgrado.

\begin{abstract}
This research shows the situation of former students from the Graduate Department of the Social Sciences and Humanities School (FCSH) at Escuela Superior Politécnica del Litoral (ESPOL), about their overall profile, assessment of education received, the relevance of the programs in the environment in which they operate, and the impact on employment, among other things. This, quantitative - descriptive approach, considered 273 former students until July 2014. The investigation was applied throughout an online questionnaire using Google drive as a tool. Conclusions drawn from this research will help to expand the information previously collected about this group of former students, in order to help take measures and present suggestions that will maintain the continuous improvement process of the academic unit.
\end{abstract}

Key Words: Graduates follow-up, graduate studies, former graduate students

\title{
Antecedentes prácticos
}

Desde el año 2007, con el gobierno del Economista Rafael Correa Delgado, el Ecuador inició una etapa de cambios y transformaciones en todos los ámbitos y la educación superior no ha sido la excepción. A partir del año 2010, con la entrada en vigencia de la Ley Orgánica de Educación Superior (LOES) y la creación de los entes reguladores, se han derivado una serie de reglamentos y procedimientos que rigen a las instituciones de educación superior (IES). El objetivo de la aplicación de la LOES es definir los principios de la educación; garantizar el derecho a la educación superior de calidad que propenda a la excelencia, al acceso universal, permanencia, movilidad y egreso de sin discriminación alguna.

Este objetivo cumple con los principios del desarrollo de la educación internacional y busca elevar la calidad del sistema ecuatoriano de educación superior. En los últimos años se han impulsado procesos de evaluación interna y externa en las instituciones de educación en todos los niveles y en diferentes ámbitos tales como: academia, eficiencia académica, investigación, organización e infraestructura (Consejo de Evaluación, Acreditación y Aseguramiento de la Calidad de la Educación Superior, 2011). Está claro el hecho de que, si el Ecuador espera competir internacionalmente, tiene que estar a la vanguardia del conocimiento y las buenas prácticas.

La LOES estipula que las instituciones de educación superior en el Ecuador deberán implementar sistemas de seguimiento a sus graduados y graduadas e informar al Consejo de Evaluación, Acreditación y Aseguramiento de la Calidad de la Educación Superior (CEAACES) sobre sus resultados. El Modelo de Evaluación Institucional aplicado por el CEAACES contempla la verificación de que las instituciones de educación superior cuenten con procesos para el seguimiento a los sujetos graduados y uso de la información de un sistema informático de seguimiento para la retroalimentación de aspectos académicos de la institución. 
Desde la entrada en vigencia de la LOES (2010), la Facultad de Ciencias Sociales y Humanísticas (FCSH) ha iniciado esfuerzos para planificar, medir y mejorar su calidad mediante procesos establecidos para una mejora continua, planteando entre las metas institucionales, el fortalecimiento de la relación que existe entre las personas graduadas de los programas de postgrados y la Facultad. Dando prioridad a este objetivo, se realizó un estudio a estudiantes que han egresado de todos los programas de los postgrados activos e inactivos de la Facultad (tabla 1), con el fin de obtener información sobre la evaluación de la enseñanza recibida, la pertinencia de los programas en el entorno en el que se desenvuelven, el impacto en su empleo (sea en nuevos puestos de trabajo o en el salario) y otros aspectos relacionados.

Tabla 1

Programas de maestrías activos e inactivos considerados en la encuesta

\begin{tabular}{l}
\hline \multicolumn{1}{c}{ Programas activos } \\
\hline Maestría en Finanzas (MFIN) \\
\hline Maestría en Economía y Dirección de Empresas (MEDE) \\
\hline Maestría en Gestión del Talento Humano (MGTH) \\
\hline Programas inactivos \\
\hline Maestría en Docencia e Investigación Educativa (MEDIE) \\
\hline Maestría en Marketing y Comercio Internacional (MMCI) \\
\hline
\end{tabular}

Nota: Elaboración propia a partir de archivos de la FCSH.

\section{Referentes conceptuales}

Existen diferentes definiciones sobre lo que representa estudiar en un programa de postgrados. De acuerdo con Manzo, Rivera y Rodríguez (2006):

La educación de posgrado es un conjunto de procesos de enseñanza-aprendizaje dirigidos a garantizar la preparación de los graduados universitarios para complementar, actualizar y profundizar en los conocimientos y habilidades que poseen, vinculados directamente al ejercicio profesional, los avances científico-técnicos y las necesidades de las entidades en que laboran. (p. 16)

Según Sánchez y Arredondo (2001), el principal objetivo de las maestrías es proporcionar a sus estudiantes formación sólida y amplia en una determinada área, con la intención de que se introduzcan en el quehacer investigativo para realizar ejercicio docente y profesional. El ritmo de cambios en los conocimientos básicos y en el plano de las exigencias sociales 
y éticas exige que todo profesional prosiga su formación (UNESCO, 1998). Según Teichler (2005), en el ámbito laboral, la educación tiene dos funciones básicas: la de preparar, otorgar competencias, destrezas, habilidades y la de distribuir el status, pues según el nivel educativo obtenido se podrá determinar el acceso a recursos monetarios y el reconocimiento social.

Por otro lado, se consideran sujetos graduados a profesionales que finalizaron con un plan de estudio y obtuvieron el perfil académico-profesional previsto, entre otros requisitos de graduación, por lo cual se les otorga una titulación (Agencia Centroamericana de Acreditación de Posgrados, 2008).

En Ecuador, el cuarto nivel o estudios de postgrados están orientados a proveer entrenamiento profesional avanzado o a la especialización científica y de investigación. Se consideran títulos de cuarto nivel a las especializaciones, maestrías y doctorados (Consejo de Educación Superior (CES), 2010). En la misma ley, se estipula que las instituciones de educación superior en el Ecuador deberán implementar sistemas de seguimiento a sus estudiantes que se han graduado e informar al CEAACES sobre sus resultados. El Modelo de Evaluación Institucional, desarrollado por este organismo de control, contempla la verificación de que las instituciones de educación superior cuenten con procesos para el seguimiento a sus sujetos graduados (CEAACES, 2011).

El manual de instrumentos y recomendaciones sobre el seguimiento de egresados del Tecnológico de Monterrey (Red GRADUA2 y Asociación Columbus, 2006) plantea que los aspectos principalmente considerados en estudios de seguimiento a personas graduadas son: Acreditación, desarrollo curricular, afiliación universitaria, instrumentos para la política social e información relevante para los grupos de interés, dentro de los cuales se encuentran directivos de instituciones educativas, comunidad universitaria y estudiantes de los diferentes programas de estudio (Jaramillo, Giraldo y Ortiz, 2006).

Lainformación obtenida de estetipo de estudios puede proveerimportanteretroalimentación para la pertinencia curricular y sus posibles adecuaciones (Simón y Arellano, 2009). También coadyuva a la elaboración de diagnósticos útiles que brinden información ampliada sobre las tendencias de ocupación, sueldo, tiempo de colocación en el mercado laboral, satisfacción con la formación recibida y la aplicación de conocimientos adquiridos en el empleo actual (Rengifo, 2013). En algunas universidades alemanas estos trabajos han servido para identificar la formación que el mercado laboral requiere (Schomburg, 2004).

Las tareas de las instituciones de educación superior deben ser pertinentes (Brünner, 2009), en el sentido de que los programas de estudio deben dar respuesta a las necesidades de la sociedad, y considerar aspectos económicos y apertura al mundo laboral (Ketele, 2008). Además, la tasa de empleabilidad impacta sobre la valoración de los resultados de la universidad; por lo cual, al tener en cuenta esta información, se puede trabajar en la actualización curricular y asegurar la pertinencia del programa que mejore la situación de quien se gradúa (Gentili, 2012), lo que llevará a que la institución también avance (Hernández, Tavera y Jiménez, 2012).

El estudio de seguimiento a sujetos graduados da la oportunidad de mejorar los procesos institucionales mediante el levantamiento, análisis de información relacionada al desempeño profesional y personal de las personas graduadas, así como contribuir a la explicación del desempeño profesional (Red GRADUA2 y Asociación Columbus, 2006). 
En aulas tradicionales, la satisfacción de sus estudiantes se da por las características de los mismos grupos de estudiantes, el tipo de relación con sus docentes, el currículo de estudios, la formación, su vida estudiantil, los servicios de apoyo, los recursos y la infraestructura (Bolliger y Martindale, 2004). Bean y Bradley (1986) concluyen que entre los mejores predictores de la satisfacción estudiantil se encuentran la integración académica, utilidad de la educación, el nivel de dificultad del programa de estudios y la calidad; este último resulta de interés primordial tanto para estudiantes, personal académico, como para responsables de la política e investigaciones de educación superior a nivel internacional (Cheng, Taylor, Williams y Tong, 2016). Desde el punto de vista de los resultados financieros de la universidad, es importante la satisfacción de los individuos graduados por las probabilidades de que vuelvan y refieran a otras personas positivamente para que realicen sus estudios en los programas que se oferten (Blackwell, Miniard y Engel, 2005).

Con base en lo expuesto, se realiza el estudio de seguimiento a sujetos graduados para mantener actualizada la base de información y su perfil, conocer su opinión sobre la satisfacción con la enseñanza recibida, la pertinencia curricular en el mercado laboral, la tasa de empleabilidad y otros aspectos para la mejora de procesos administrativos e infraestructura. Se espera que la información recabada permita conocer si los programas de estudio lograron responder a parte de las necesidades para las cuales fueron diseñados e implementados. A su vez, los resultados permitirán tomar decisiones para la implementación de mejoras, según las debilidades identificadas y fortalezas del programa de estudio (Jorquera, 2006).

\section{Procedimientos metodológicos}

Para dar inicio al presente estudio, se debió realizar la actualización de datos de la totalidad de sujetos graduados de los seis programas de postgrado citados en la tabla 1 para el periodo 1998-2014. Esta tarea fue realizada mediante un trabajo conjunto con el Departamento Integra ${ }^{3}$ de la Facultad y el personal del Departamento de Postgrado, quienes se encargaron de dicha actualización a través de contacto telefónico y mediante correo electrónico. Al finalizar esta etapa, se estimó una muestra clasificada por estratos.

Entre agosto y septiembre de 2014, se les convocó mediante correo electrónico para realizarles la encuesta diseñada para este estudio, la cual fue aplicada mediante la herramienta de Google Drive.

La encuesta estuvo dividida en cinco secciones para analizar diferentes aspectos de los sujetos graduados. De un total de 35 preguntas, 9 eran socioeconómicas y aspectos generales, 5 estaban relacionadas con el impacto en el empleo, 6 se destinaron para conocer la pertinencia de estos, 12 para conocer sobre la relación entre las personas graduadas y la facultad y 3 para conocer sobre la contribución de la educación recibida. El tiempo de recolección de datos fue de aproximadamente 30 días, y la tasa de respuesta fue de $21 \%$.

A continuación, se muestran los resultados de las distintas secciones, con el análisis descriptivo y gráfico de estas.

3 El Departamento Integra es un programa de inserción laboral para estudiantes y personas egresadas de los programas de pregrado y postgrados de la FCSH. 


\section{Análisis y discusión de los resultados}

El cuestionario se envió a los 273 individuos graduados de los diferentes programas de maestrías, de los cuales se obtuvo una tasa de respuesta del $21 \%$. Se recibió un porcentaje de $1.75 \%$ de respuestas de la Maestría en Docencia e Investigación Educativa, un 24.56\% de respuestas de la Maestría en Economía y Dirección de Empresas, un 12.28\% de respuestas fueron de la Maestría en finanzas, un $26.32 \%$ de la Maestría en Talento Humano y, por último, el mayor porcentaje de respuestas fue de estudiantes de la Maestría en Marketing y Comercio Internacional con un 35.09\%.

\section{Perfil general de estudiantes que han egresado}

Del $21 \%$ de individuos graduados que contestaron la encuesta, aproximadamente el $52.63 \%$ son hombres, mientras que el $47.37 \%$ son mujeres, con una edad promedio de 36 años. En cuanto a su ocupación, el $10.53 \%$ son personas empresarias, mientras que el restante $89.47 \%$ son personal empleado o ejecutivo de empresas.

Por otro lado, en cuanto a su profesión, se pudo observar que existen muchos campos en su titulación de tercer nivel, como se demuestra en la figura 1. Esto se da debido a que los programas de postgrado que ofrece el departamento son muy amplios en cuanto a la formación de estudiantes, por lo que históricamente se ha contado con estudiantes con títulos desde ingeniería agronómica, hasta contaduría pública autorizada.

\section{¿Cuál es su profesión?}

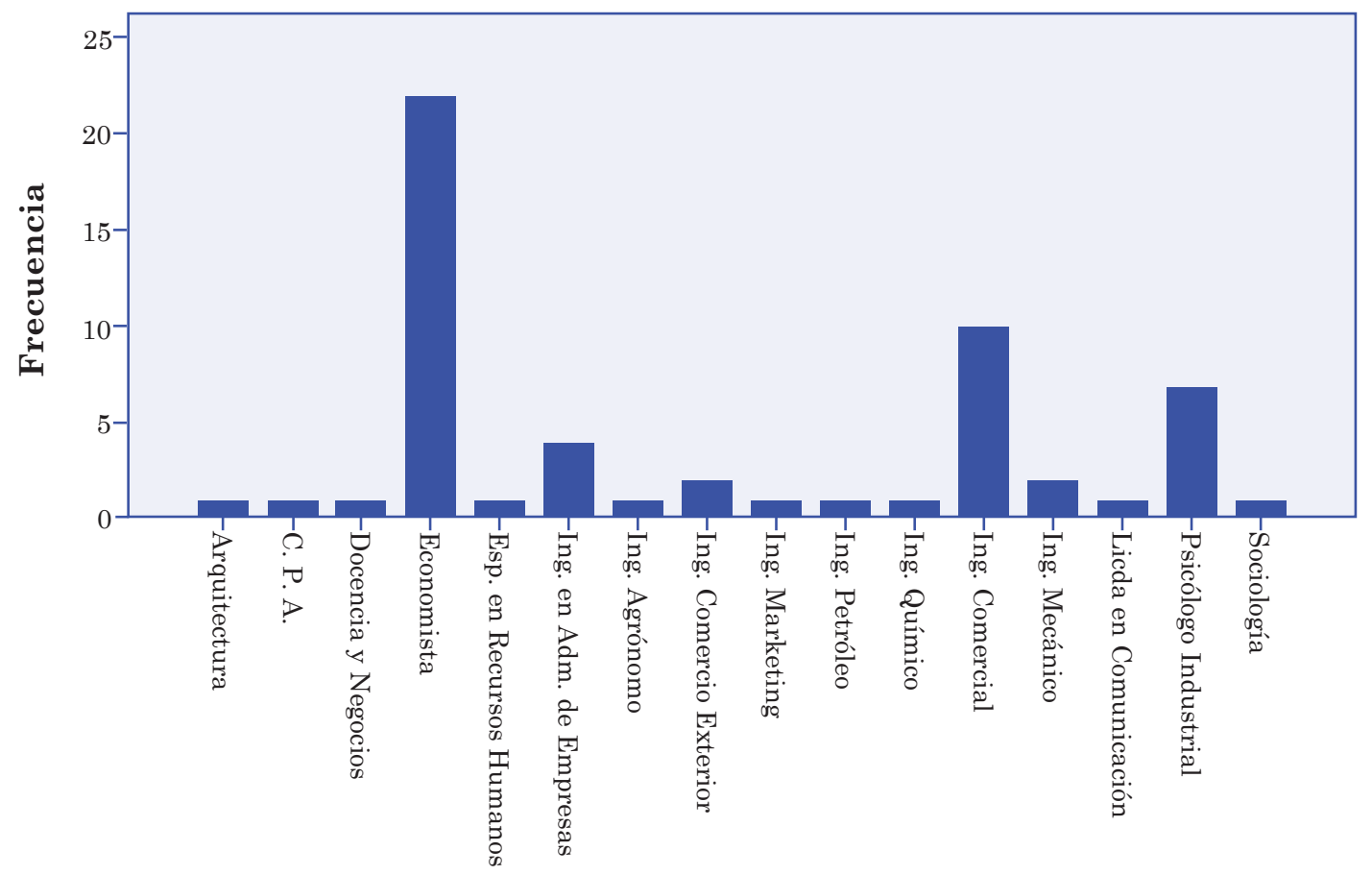

Figura 1. Profesión de sujetos graduados de Postgrado de la FCSH. Elaboración propia a partir de las encuestas realizadas. 
La experiencia laboral promedio de los sujetos encuestados es de 13 años, y el promedio de experiencia gerencial de 4 . En la figura 2 se muestra que aproximadamente el $40.35 \%$ del total tiene una experiencia laboral de entre 3 a 9 años, mientras que el $63.16 \%$ cuenta con experiencia gerencial de entre 0 a 4 años.
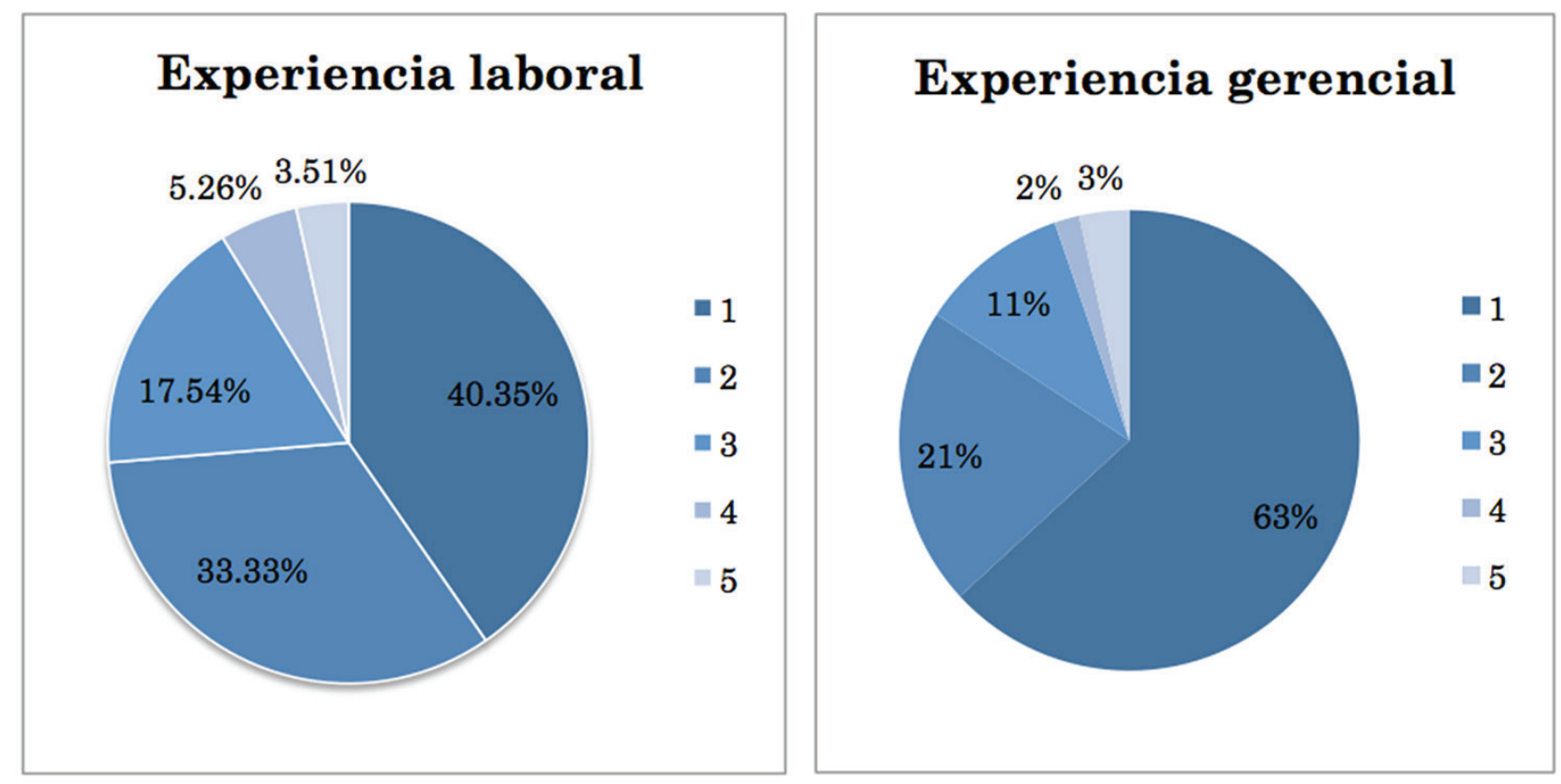

Figura 2. Experiencia laboral y gerencial de personas graduadas de Postgrado. Elaboración propia a partir de las encuestas realizadas.

Aproximadamente un 57.9\% de los sujetos graduados encuestados se emplea en empresas privadas y un $31.6 \%$ labora en empresas públicas, mientras que el 10.5\% cuenta con empresas propias (ver Figura 3).

Los sectores de la empresa donde laboran, mayoritariamente pertenecen a industrias manufactureras, con un porcentaje de $15.79 \%$, mientras que $12.28 \%$ pertenecen al sector de enseñanza, y otro $12.28 \%$ a los sectores de agricultura, ganadería, silvicultura y pesca.

\section{La pertinencia curricular en el mercado laboral}

La pertinencia se relaciona con las necesidades de la sociedad, especialmente en su aspecto productivo, pretende adecuar lo que se ofrece desde la educación formal a la demanda potencial del mercado laboral. Por eso se planteó un grupo de preguntas vinculadas al tema.

En la figura 4 se observa que el 52.63\% de las personas entrevistadas están de acuerdo con que el programa de Maestría tiene vinculación con el entorno local y regional y un $49.12 \%$ con el entorno global, como se muestra en la figura 5. 


\section{Sectores laborales}

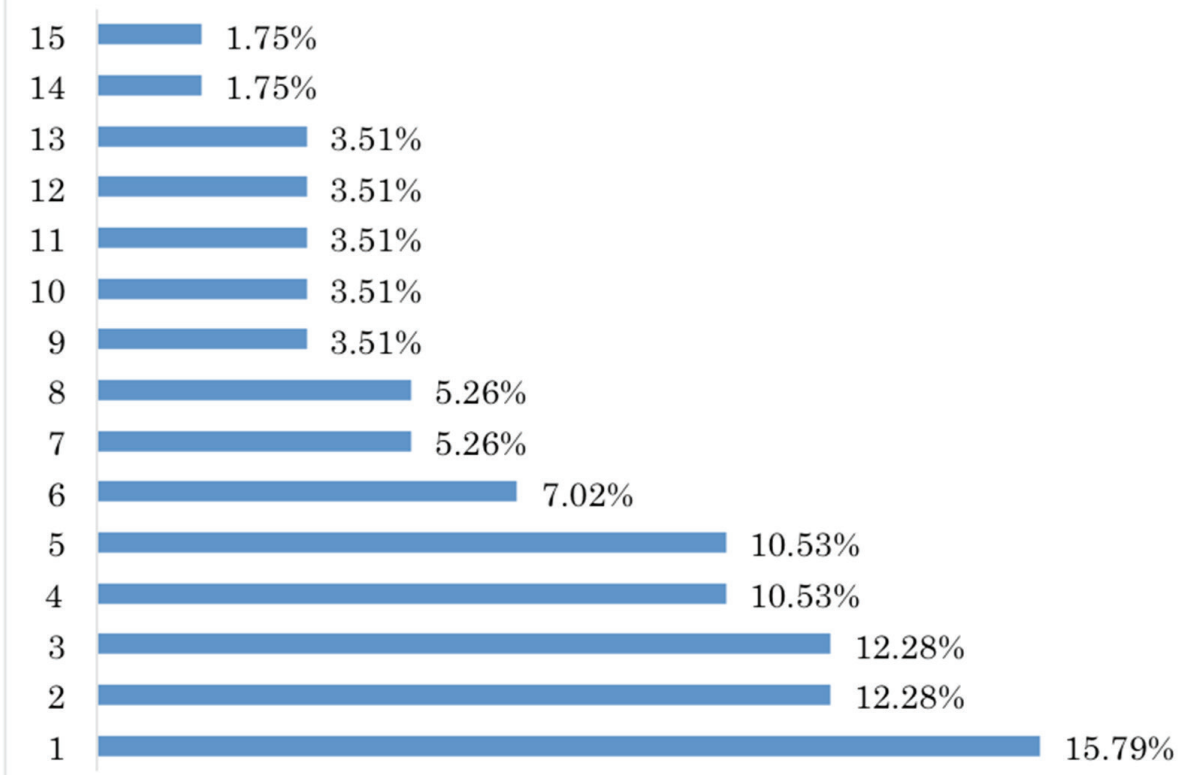

Figura 3. Sectores laborales donde trabajan las personas graduadas de Postgrados. Elaboración propia a partir de las encuestas realizadas.

\section{Vinculación con el entorno local y regional}

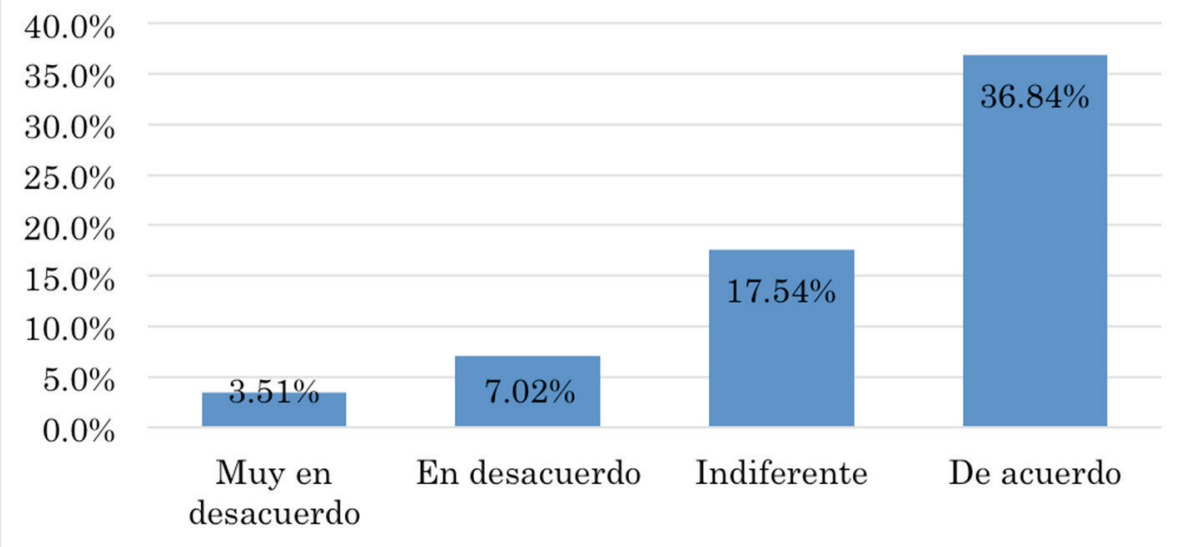

Figura 4. Conformidad con la vinculación en el entorno local. Elaboración propia a partir de las encuestas realizadas. 


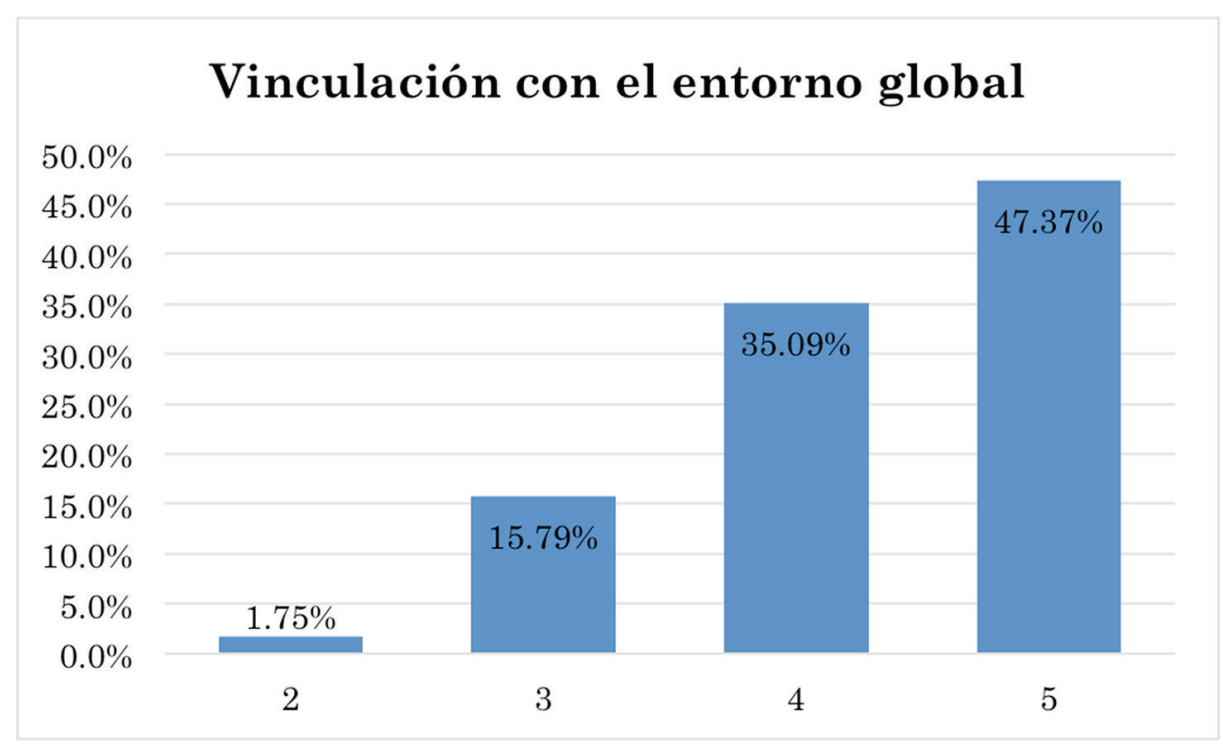

Figura 5. Conformidad con la vinculación en el entorno global.

Elaboración propia a partir de las encuestas realizadas.

En la figura 6 se observa que aproximadamente el 40.35\% del grupo egresado encuestado está de acuerdo con que el programa de Maestría ha producido un impacto significativo en el desarrollo social en el área de influencia.

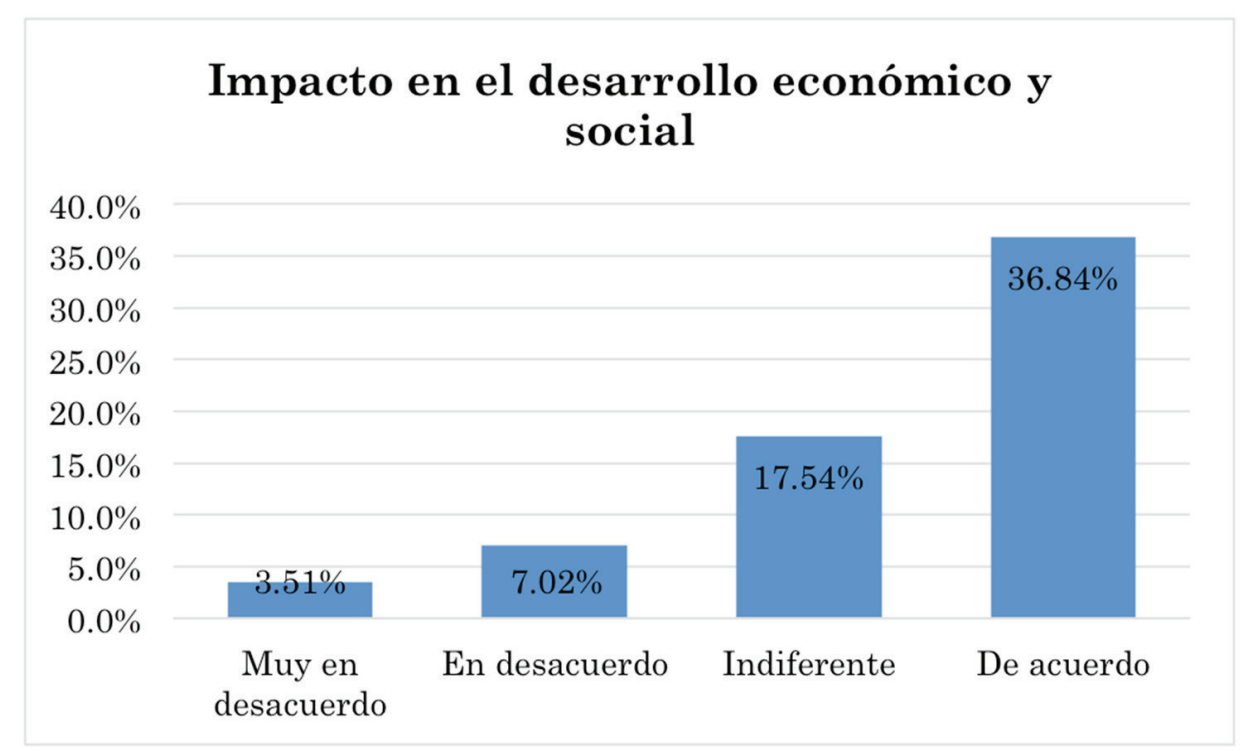

Figura 6. Conformidad con el impacto económico y social de la Maestría. Elaboración propia a partir de las encuestas realizadas. 


\section{La satisfacción en las personas graduadas por la enseñanza recibida}

En la figura 7 se refleja que el $42.11 \%$ de las personas graduadas encuestadas de las Maestrías están muy de acuerdo con que el programa de Maestría tuvo una incidencia positiva en la adquisición de nuevas competencias de pensamiento crítico.

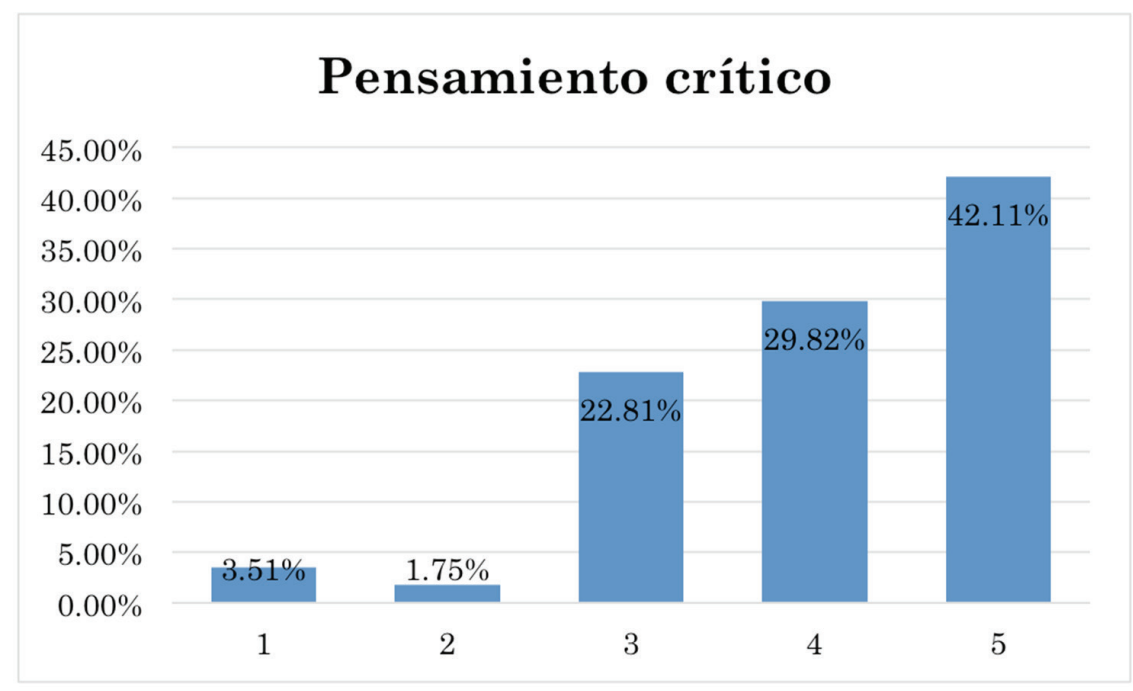

Figura 7. Adquisición de competencias en pensamiento crítico. Elaboración propia a partir de las encuestas realizadas.

Aproximadamente el 38,60\% estuvo de acuerdo con que el programa de Maestría tuvo alta incidencia en la adquisición de competencias en el trabajo en equipo y el 43,86\% está de acuerdo con que ha obtenido nuevas competencias en la toma de decisiones, tal como se muestra en la figura 8 y 9.

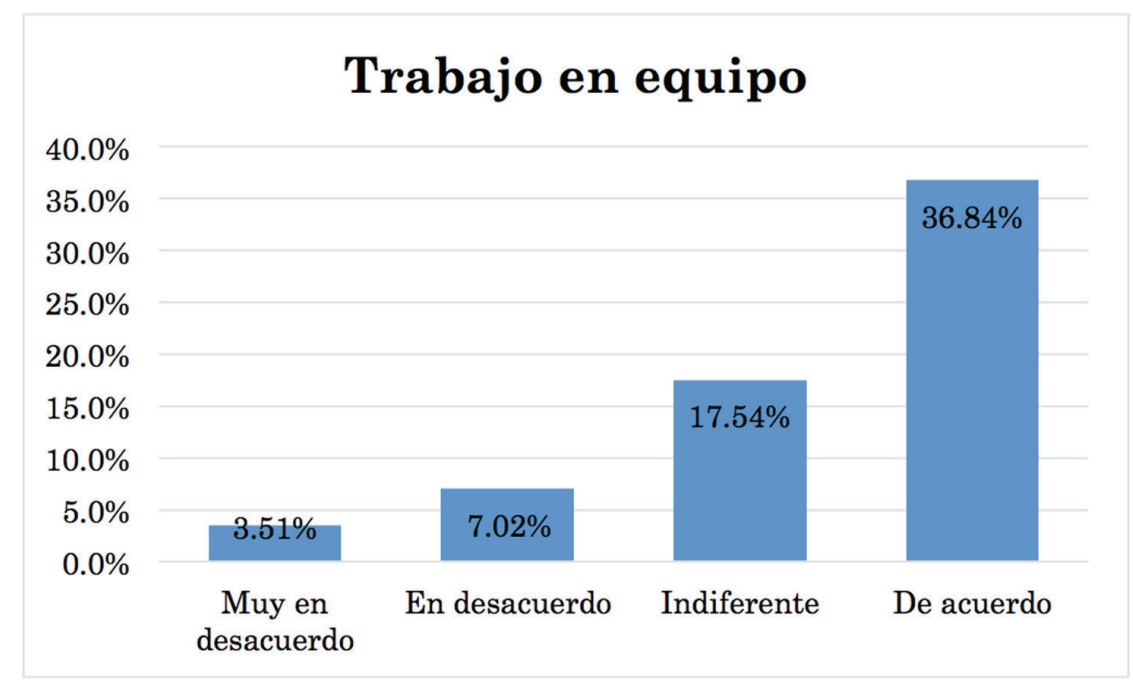

Figura 8. Adquisición de competencias en trabajo en equipo.

Elaboración propia a partir de las encuestas realizadas. 


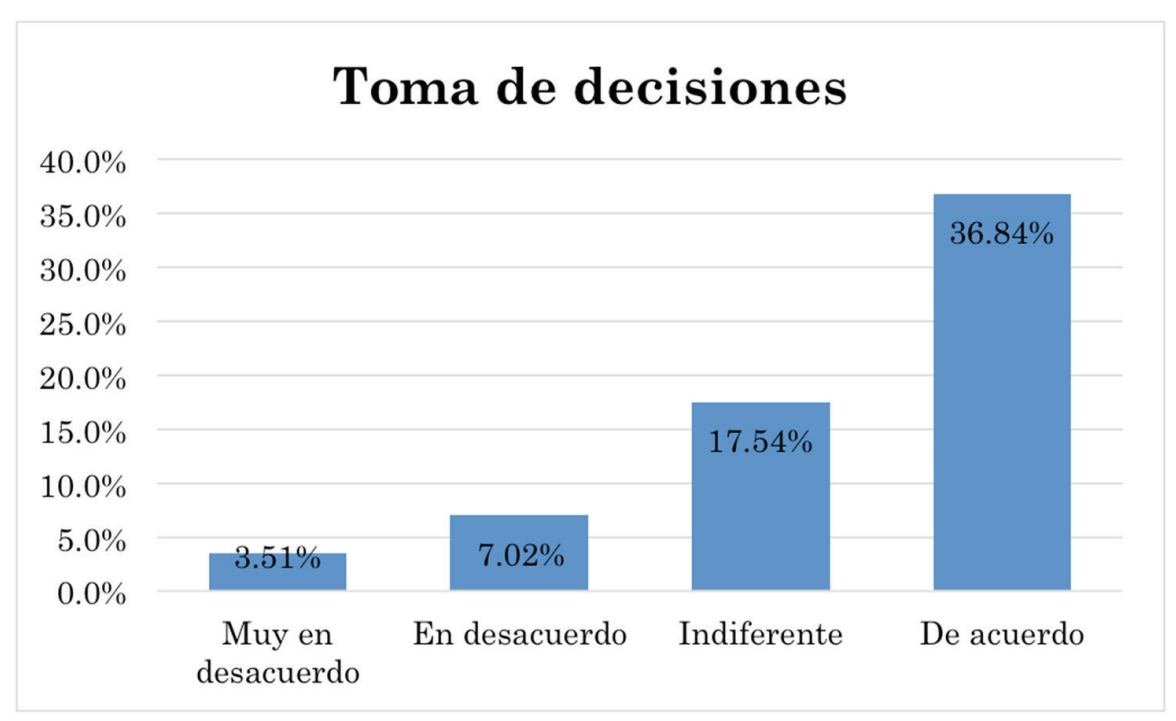

Figura 9. Adquisición de competencias en la toma de decisiones.

Elaboración propia a partir de las encuestas realizadas.

El 56,14\% estuvo de acuerdo con que el programa de Maestría tuvo alta incidencia en la adquisición de competencias con respecto a la sensibilidad global (Figura 10).

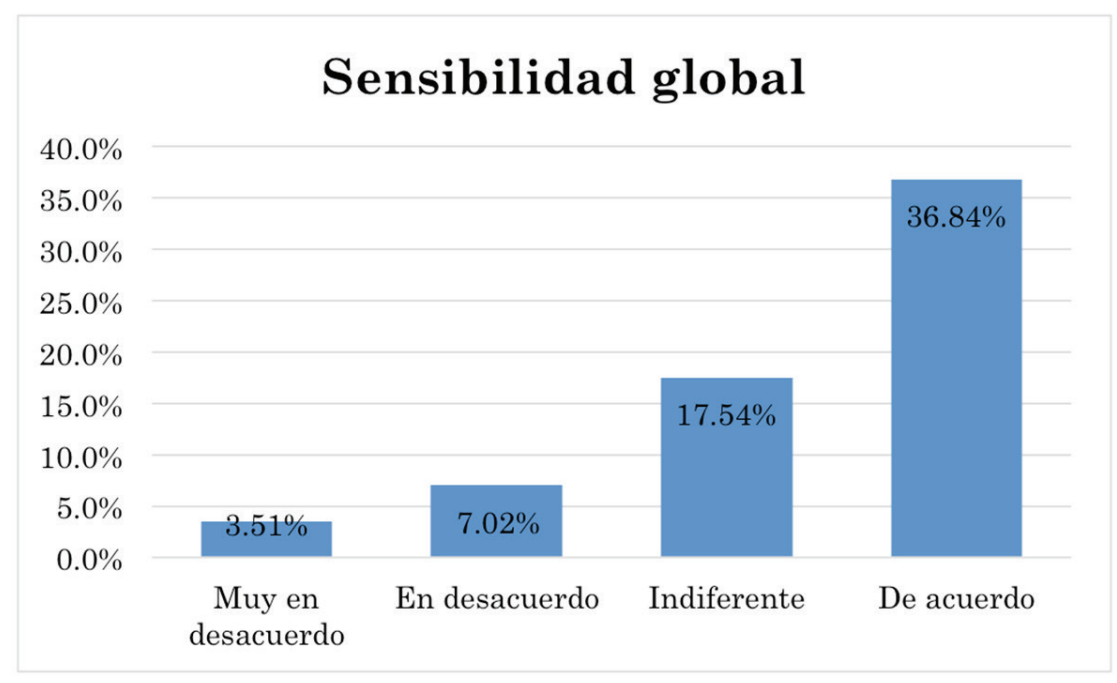

Figura 10. Adquisición de competencias con respecto a la sensibilidad global. Elaboración propia a partir de las encuestas realizadas.

\section{La tasa de empleabilidad}

Al cursar el programa de Maestría un $61.40 \%$ de los sujetos graduados encuestados no recibió un ascenso en sus puestos de trabajo. Sin embargo, el $63.16 \%$ obtuvo una mejor oportunidad de empleo. Mientras que el 43.86\% sí logró un incremento salarial (en un 25\% aproximadamente, según las respuestas de las encuestas) (Ver Figura 11). 

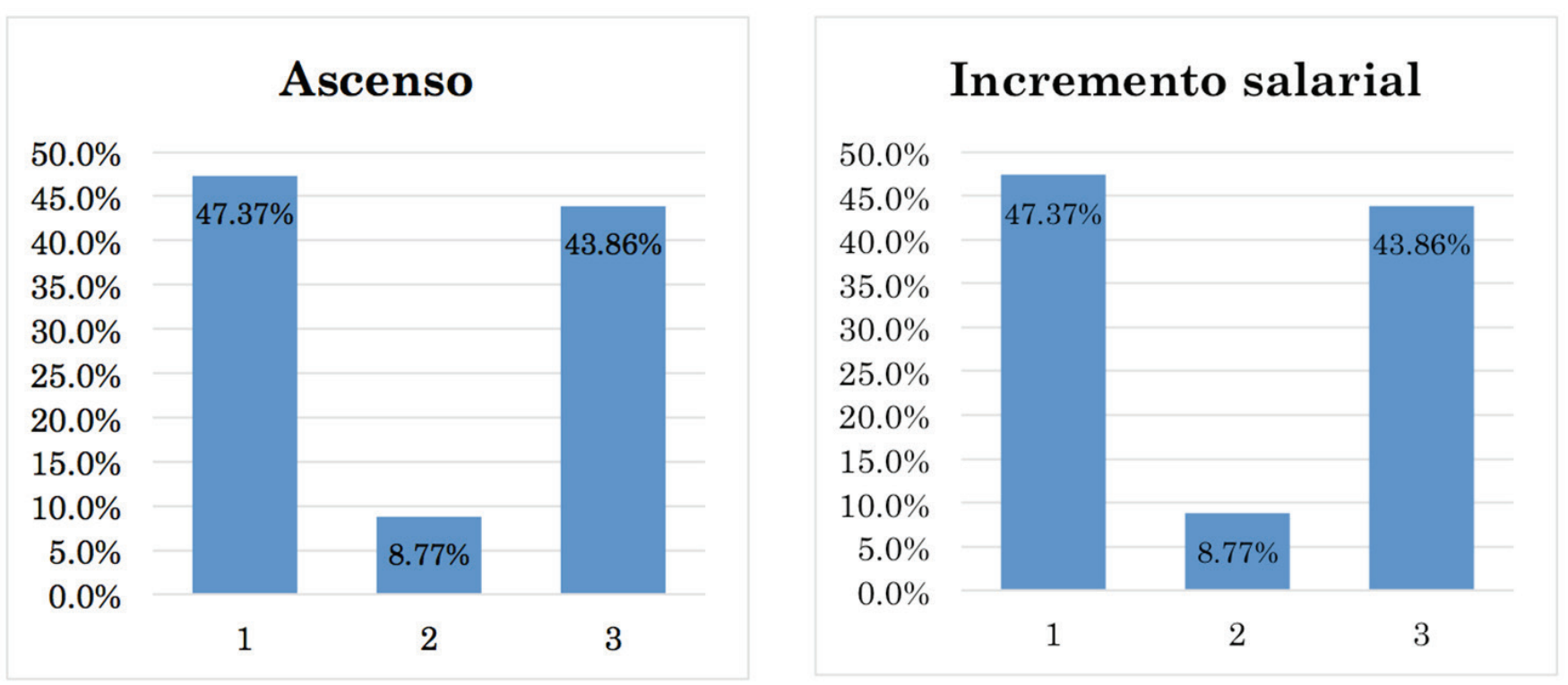

Figura 11. Ascenso vs. incremento salarial de los sujetos graduados. Elaboración propia a partir de las encuestas realizadas.

\section{Otros aspectos para mejora de procesos administrativos e infraestructura}

En este proceso de seguimiento se vinculó, además, la opinión sobre aspectos relevantes para tomarlos en cuenta en el proceso de mejora continua. En la figura 12 se observa que aproximadamente el $40.35 \%$ de los sujetos encuestados está de acuerdo en que los programas de Maestría definen y aplican en forma clara las políticas que determinan la permanencia, promoción y graduación del estudiantado, mientras que el 33.33\% respondió que están muy de acuerdo.

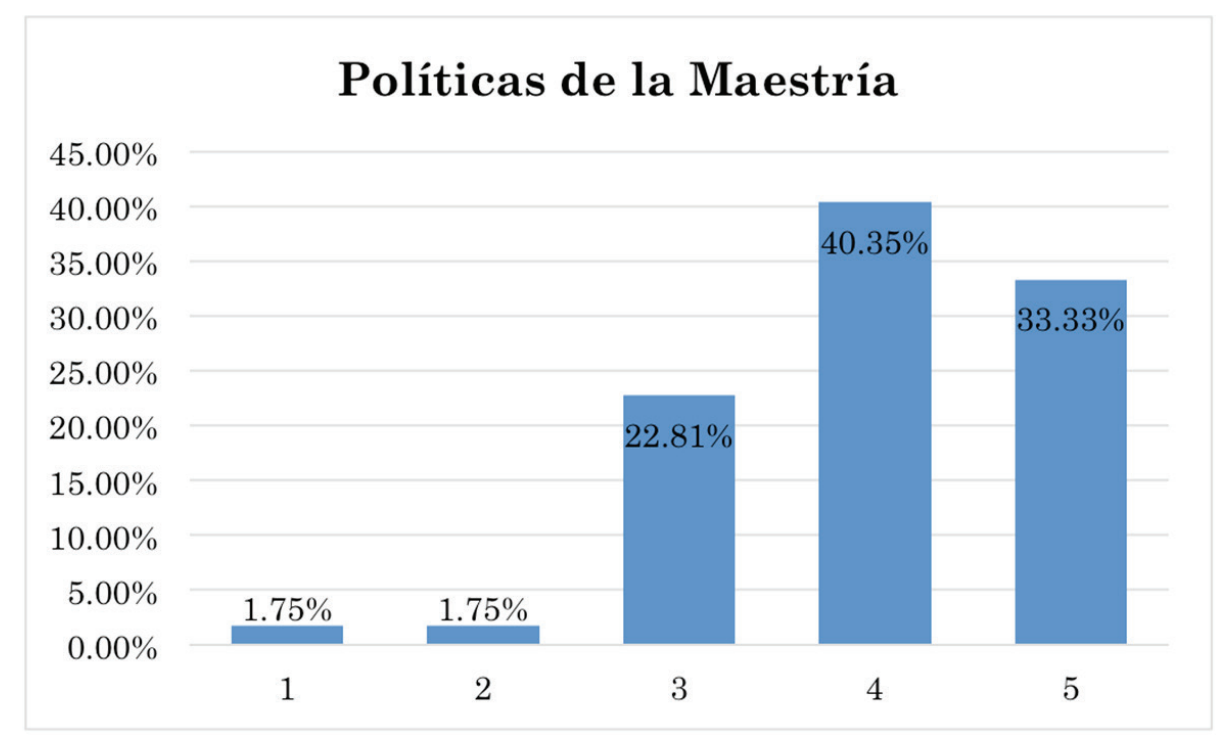

Figura 12. Conformidad con las políticas de la Maestría.

Elaboración propia a partir de las encuestas realizadas. 
Aproximadamente el $36.84 \%$ de sujetos egresados está de acuerdo en que la FCSH difunde la información sobre las actividades de la Maestría de forma oportuna (figura 13).

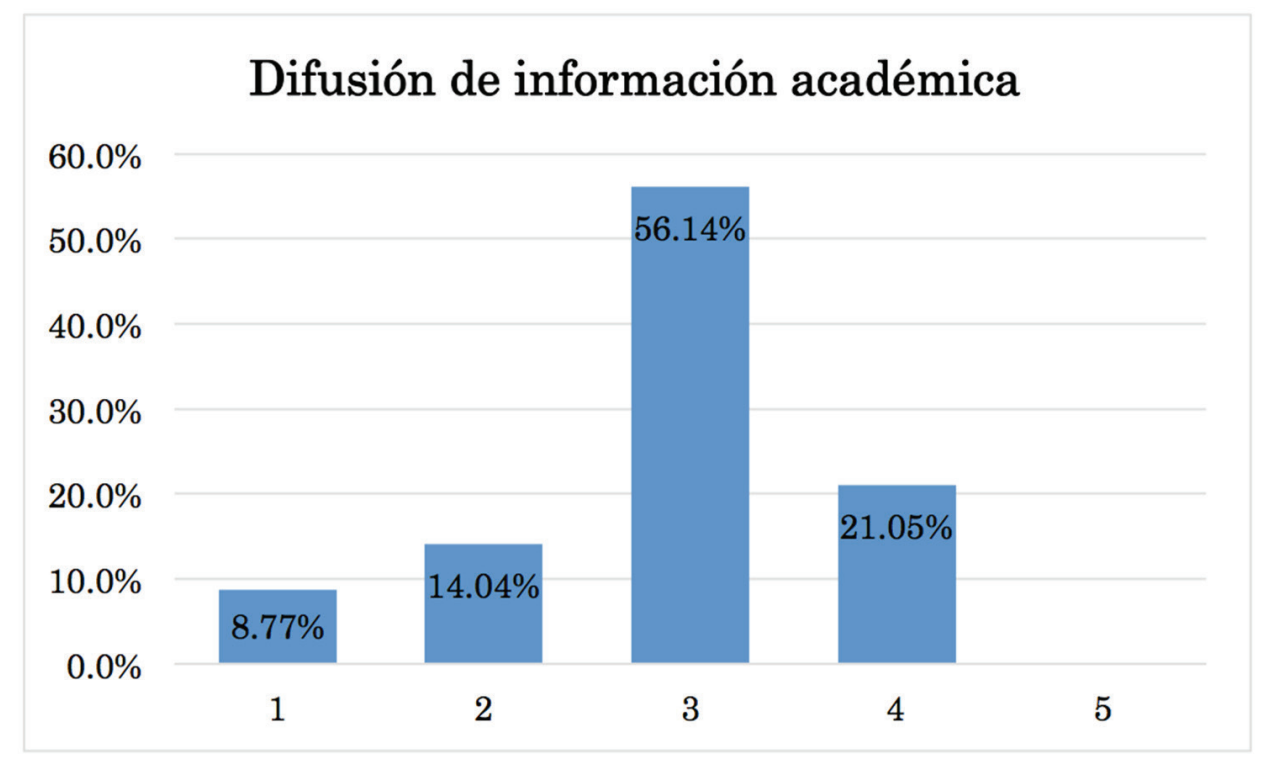

Figura 13. Conformidad con la difusión de información de la Maestría.

Elaboración propia a partir de las encuestas realizadas.

Con respecto a la relación que existe entre los individuos graduados y la FCSH, un $38.60 \%$ estuvo de acuerdo en que la comunicación que existe entre ellos y el departamento administrativa de la Facultad es buena (figura 14).

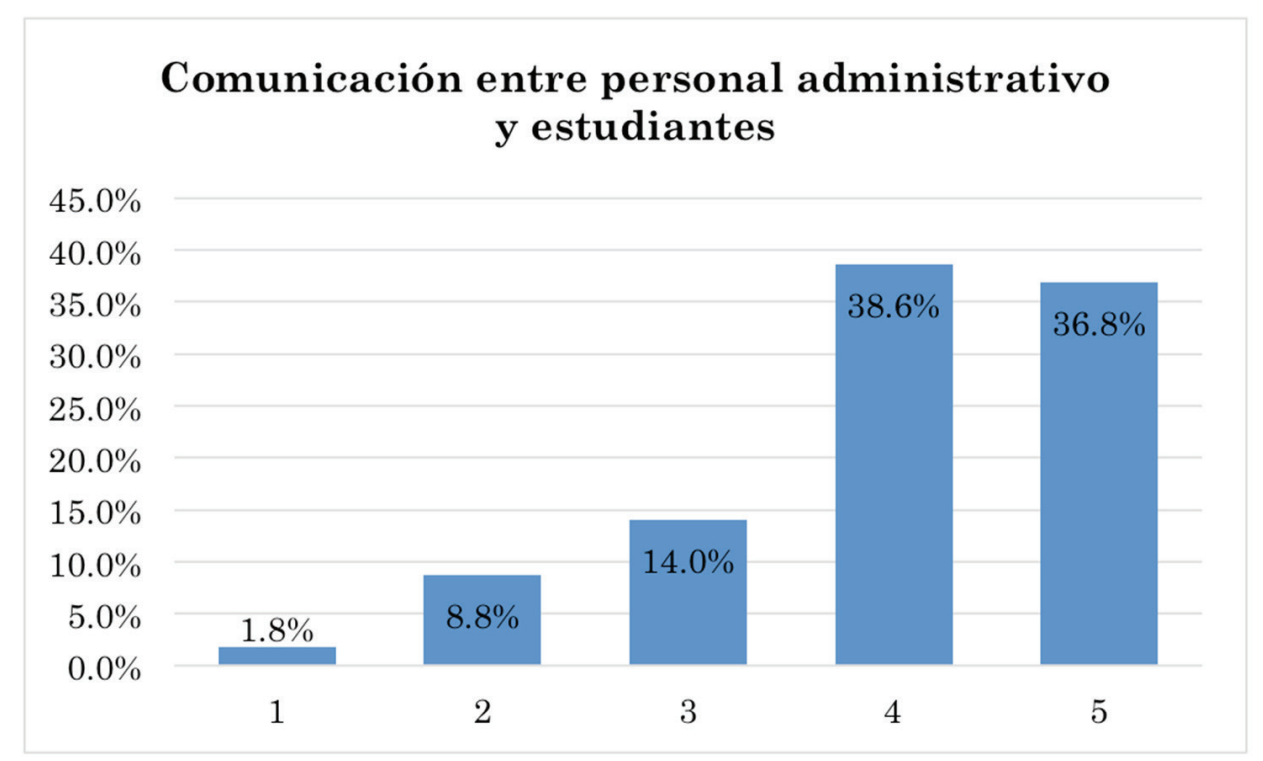

Figura 14. Comunicación entre personal administrativo y estudiantes. Elaboración propia a partir de las encuestas realizadas. 
Aproximadamente el $45.61 \%$ afirmó su acuerdo con el proceso de gestión financiera en cuanto al registro y las opciones de pago (figura 15).

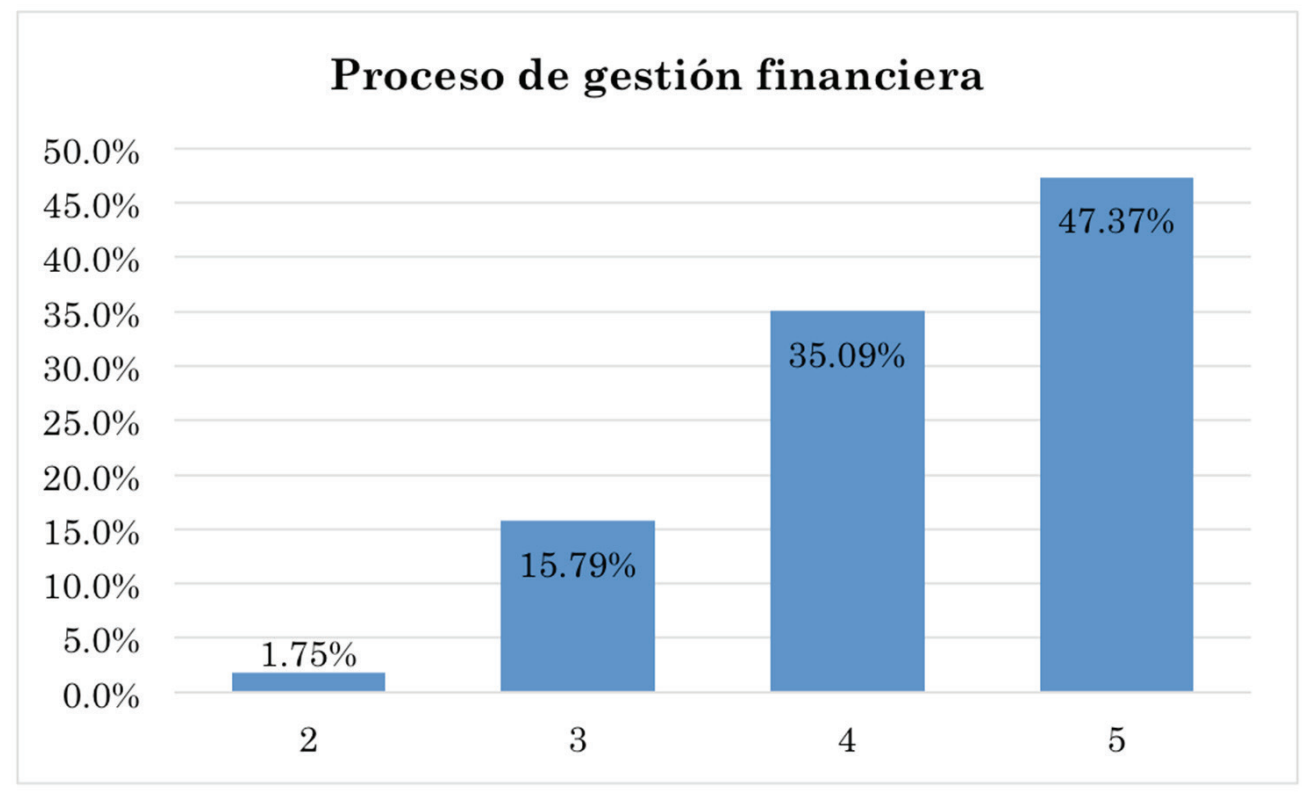

Figura 15. Gestión financiera de la FCSH.

Elaboración propia a partir de las encuestas realizadas.

El 47.37\% estuvo muy de acuerdo en que las instalaciones de la Facultad son las adecuadas para recibir clases. A la presente fecha las aulas están siendo perfeccionadas con el proceso de renovación de infraestructura en el que se encuentra la FCSH (figura 16).

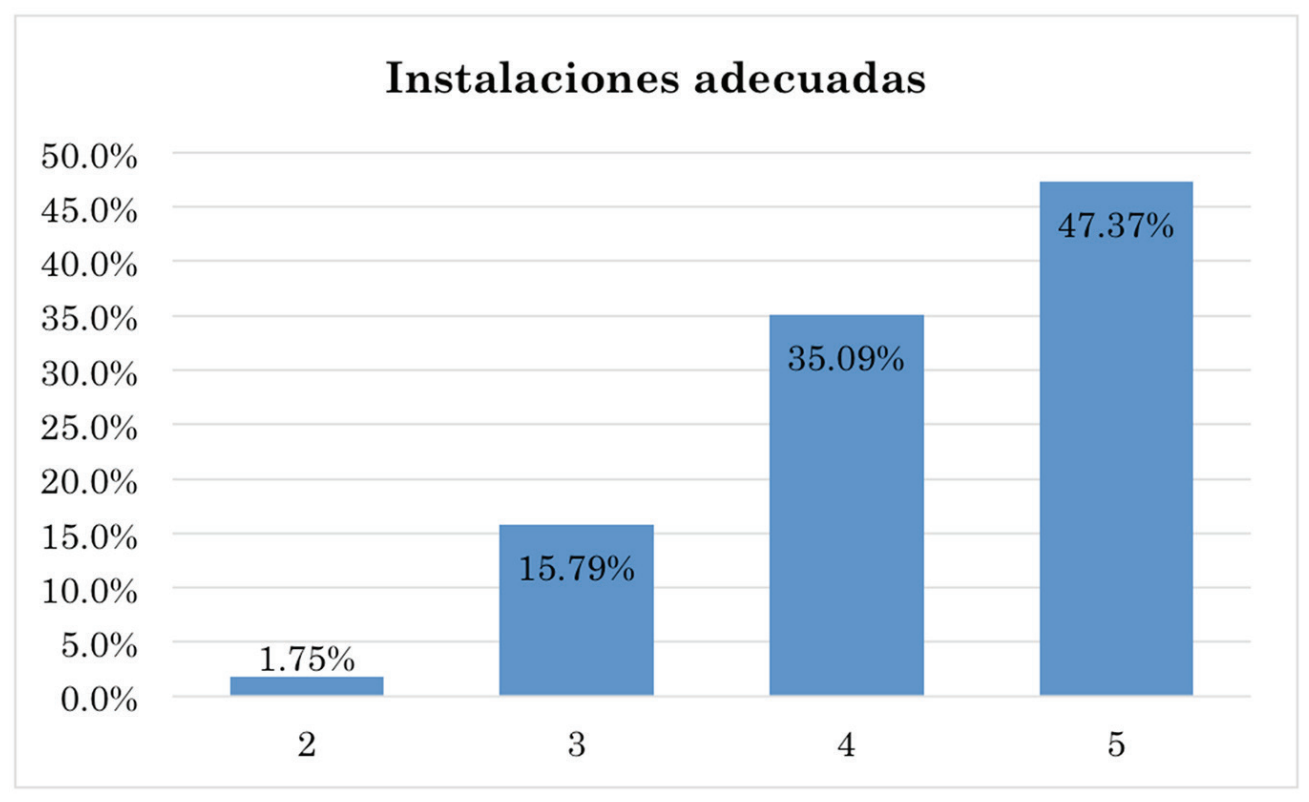

Figura 16. Calidad en las instalaciones de las aulas.

Elaboración propia a partir de las encuestas realizadas. 
Las personas graduadas encuestadas se mostraron un $43.86 \%$ muy de acuerdo (figura 17), en que el tiempo previsto para la entrega del título de Magíster se realiza en un tiempo igual o menor a lo establecido (12 meses).

\section{Tiempo para completar la titulación}

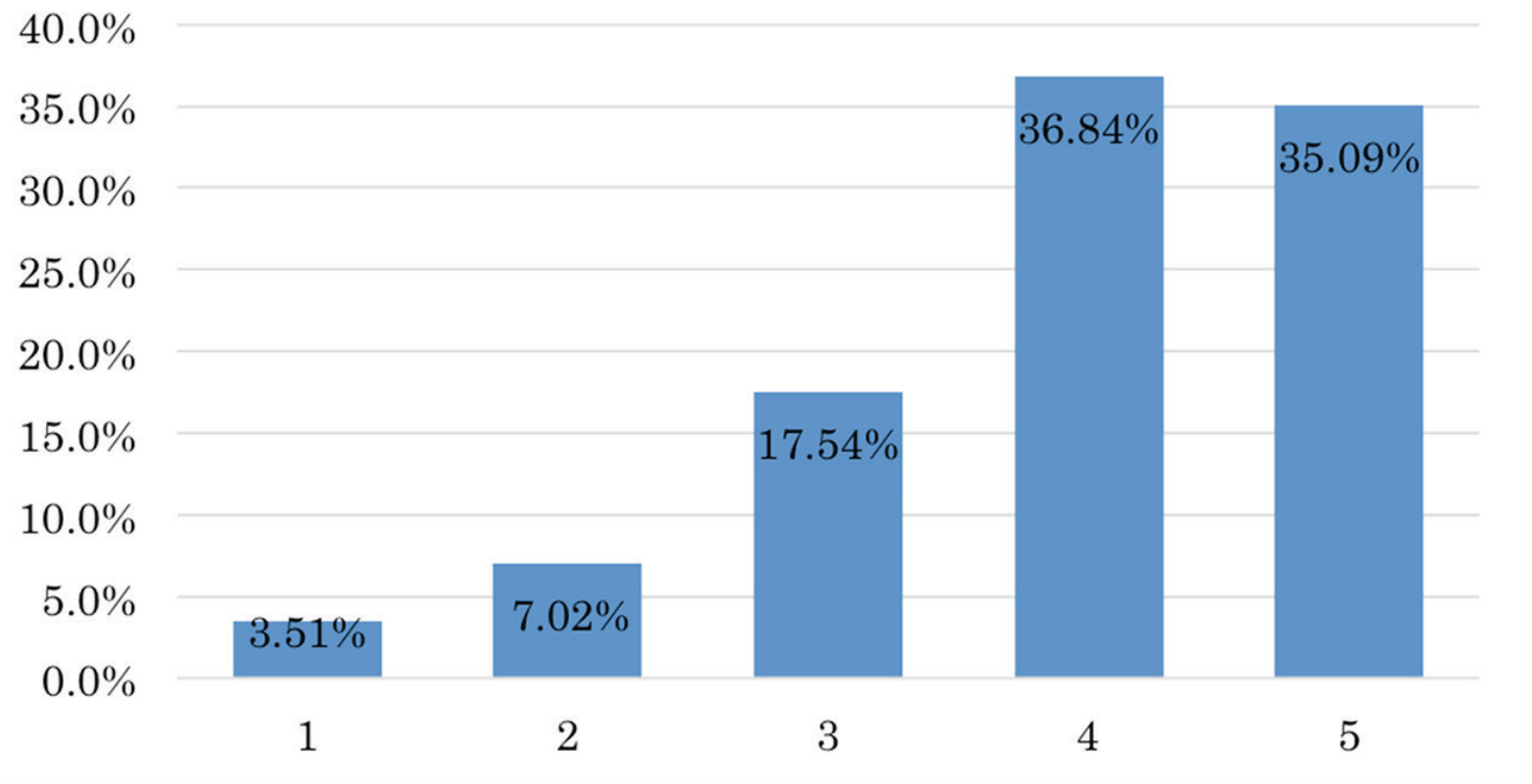

Figura 17. Tiempo para completar la titulación.

Elaboración propia a partir de las encuestas realizadas.

El 83\% indicó haber sido contactado por la Facultad después de su graduación. El 86\% han recibido invitaciones a los eventos que la Facultad ha realizado en los últimos 12 meses. Y al 88\% le interesaría formar parte de la Asociación de Exalumnos de Postgrados de la Facultad.

$\mathrm{Al}$ consultarles acerca de su conocimiento de la página web http://www.fcsh.espol.edu.ec/, el $52.63 \%$ la visita muy pocas veces y el $82,45 \%$ no la conocen o no han leído las publicaciones que la Facultad realiza; sin embargo, el 57.89\% sí han recibido la revista informativa de la Facultad llamada Noti.

Además, tal como es posible observar en la tabla 2, el 24.6\% indicó que al registrarse en el programa, su principal fuente de información fue el periódico. 
Tabla 2

Fuente de información utilizadas por los individuos graduados encuestados de Postgrados

\begin{tabular}{cc}
\hline Fuente & $\begin{array}{c}\text { \% de estudiantes } \\
\text { que han egresado }\end{array}$ \\
\hline Colegas/amistades & $19,3 \%$ \\
E-mail de Promoción del Programa & $19,3 \%$ \\
Ex alumnos/alumnos de ese entonces & $15,8 \%$ \\
Llamada del equipo de comercialización & $1,8 \%$ \\
Página web de la FCSH & $14,0 \%$ \\
Periódico & $24,6 \%$ \\
Revista & $1,8 \%$ \\
Otros & $3,4 \%$ \\
\hline
\end{tabular}

Nota: Elaboración propia a partir de archivos de la FCSH.

\section{Conclusiones y recomendaciones}

De acuerdo con la información levantada, la mayoría de estudiantes con quienes ha contado el Departamento de Posgrado en el período estudiado son hombres, con edad promedio de 36 años, 13 años en promedio de experiencia laboral y en gran medida trabajadores y trabajadoras del sector privado.

Menos del 50\% de los sujetos encuestados considera que el programa de estudios tuvo una incidencia positiva en su adquisición de nuevas competencias de pensamiento crítico, trabajo en equipo y toma de decisiones. Una cantidad importante recibió una mejor oportunidad laboral y una parte, un incremento salarial, sin que esto pueda generalizarse.

Menos del 50\% de las personas encuestadas considera que el programa tuvo vinculación con el entorno global, impacto importante en el desarrollo social. La mayoría siente satisfacción con los servicios recibidos de la FCSH.

Para continuar con el proceso de mejora continua, es importante tomar acciones sobre los puntos críticos identificados en este levantamiento de información en relación con cada programa, tal como la vinculación con el entorno, impacto en el área de influencia, desarrollo de competencias relacionadas con el pensamiento crítico, trabajo en equipo, toma de decisiones y sensibilidad global, mejora en los procesos administrativos de la unidad académica, entre otros.

Se recomienda realizar anualmente este estudio para contar con información para la mejora continua de los programas; evaluar el nivel de satisfacción de sus graduados y graduadas con la formación recibida; evaluar la pertinencia y la calidad de los planes de estudios, para la implementación de las mejoras correspondientes y para satisfacer las necesidades de los sectores empleadores; conocer el nivel de empleabilidad de las personas graduadas; conocer sobre su percepción en relación con los procesos administrativos e infraestructura, y conservar la buena comunicación entre la unidad académica y estudiantes que se graduaron en ella. 


\section{Referencias}

Agencia Centroamericana de Acreditación de Posgrados (ACAP). (2008). Manual de Acreditación ACAP. Honduras: Ideas Litográficas.

Bean, J. P. y Bradley, R. K. (1986). Untangling the satisfaction performance relationship form college students. Journal of Higher Education, 57, 393-412. doi: http://dx.doi. org/10.2307/1980994

Blackwell, R., Miniard, P. y Engel, J. (2005). Consumer Behaviour (10 ${ }^{\text {th }}$ ed). California: Southwestern College Pub.

Bolliger, D. y Martindale, T. (2004). Key factors for determining student satisfaction in online courses. International Journal on E-learning, 3(1), 61-67.

Brünner, J. J. (2009). El proceso de la educación. Estados Unidos: Harvard University Press.

Cheng, M., Taylor, J., Williams, J., y Tong, K. (Marzo, 2016). Student satisfaction and perceptions of quality: testing the linkages for $\mathrm{PhD}$ students. Higher Education Research and Development, 1-14. doi: http://dx.doi.org/10.1080/07294360.2016.1160873

Consejo de Educación Superior (CES). (2010). Ley Orgánica de Educación Superior (LOES). Gaceta del CES. Recuperado de http://www.ces.gob.ec/gaceta-oficial/reglamentos

Consejo de Evaluación, Acreditación y Aseguramiento de la Calidad de la Educación Superior (CEAACES) (2011). Modelo de evaluación. Recuperado de http://www.ceaaces.gob.ec/ sitio/modelo-generico-de-carreras-presenciales-y-semipresenciales/

Ketele, J. M. (2008). La pertinencia social de la educación superior. En Global University Network for Innovation (Coord.), Educación Superior: Nuevos retos y roles emergentes para el desarrollo humano y social. (pp. 55-59). Madrid: Mundi-Prensa.

Enciso, M. I. y Flores, J. A. (2003). Escenarios del mercado laboral Estudiantes de Posgrado. Recuperado de http://portalsej.jalisco.gob.mx/posgrado/sites/portalsej.jalisco. gob.mx.posgrado/files/pdf/escenarios mercado enciso.pdf

Flores, E. (1982). El desarrollo de la ciencia y la tecnología en la actualidad. Ciencia y Desarrollo, 8, 107-111.

Gentili, P. (2012). La persistencia de las desigualdades de género. Cuadernos del pensamiento crítico latinoamericano 52. Recuperado de http://biblioteca.clacso.edu.ar/ar/libros/secret/ cuadernos/52/52-Gentili.pdf

Hernández, C., Tavera, M., y Jiménez, M. (2012). Seguimiento de egresados en tres programas de maestría en una escuela del Instituto Politécnico Nacional en México. Formación Universitaria, 5(2), 41-52. doi: http://dx.doi.org/10.4067/S0718-50062012000200006 
Jaramillo, A., Giraldo, A. y Ortiz, J. (2006). Estudios sobre egresados la experiencia de la universidad EAFIT. Revista Universidad EAFIT, 42(141), 111-124.

Jorquera, M. L. (2006). La evaluación de programas educativos en Utemvirtual. Recuperado de http://www.utemvirtual.cl/nodoeducativo/wp-content/uploads/2006/09/loreto.pdf

Manzo Rodríguez, L, Rivera Michelena, C. y Rodríguez Orozco, A. (2006). La educación de posgrado y su repercusión en la formación del profesional iberoamericano. Educación Médica Superior, 20(3). Recuperado de http://scielo.sld.cu/scielo.php?script=sci arttext\&pid=S0864-21412006000300009\&lng=es\&tlng=es

Red GRADUA2 y Asociación Columbus (2006). Manual de instrumentos y recomendaciones sobre el seguimiento de egresado. Monterrey: Programa Editorial del Tecnológico de Monterrey y Noriega Editores. Recuperado de https://www.almalaurea.it/sites/ almalaurea.it/files/docs/universita/altro/red_gradua2.pdf

Rengifo, M. (2013). La calidad y la evaluación como estrategia de mejora y cambio en la sociedad del conocimiento: Análisis de casos en instituciones de educación superior en Europa, América del Norte y América Latina. Global Conference on Business and Finance Proceedings, 8(1), 748-752.

Sánchez, R. y Arredondo, M. (2001). Pensar el posgrado. La eficiencia terminal en ciencias sociales y humanidades de la UNAM. México: Plaza y Valdés Editores.

Schomburg, H. (2004). Manual para estudios de seguimiento de graduados universitarios. Honduras: Serviprensa.

Simón, J. D. y Arellano, J. (2009). Calidad profesional del técnico superior universitario en administración. Revista Electrónica Actualidades Investigativas en Educación, 9(2), 1-30. Recuperado de http://www.redalyc.org/articulo.oa?id=44713058007

Teichler, U. (2005). Graduados y empleo: Investigación, metodología y resultados, los casos de Europa, Japón, Argentina y Uruguay. Buenos Aires, Argentina. Facultad de Filosofía y Letras. Universidad de Buenos Aires: Editorial Miño y Dávila.

UNESCO (1998). La contribución de la educación superior al sistema educativo en su conjunto. La Educación Superior en el siglo XXI. Conferencia Mundial Sobre la Educación Superior. París, Francia. Recuperado de http://www.unesco.org/education/ educprog/wche/principal/system-s.html 